\title{
Pupillometric and personality test score differences of female aggressing pedophiliacs and normals
}

\author{
ROBERT W. ATWOOD* and ROBERT J. HOWELL \\ Brigham Young University, Provo, Utah 84601
}

A group of 10 incarcerated female-aggressing pedophiliacs was compared with a control group of inmates by their responses to a sexual deviancy scale and by pupillometrics. It was found that on the sexual deviance scale, using a cutting score of 43 , only one individual out of the 20 involved was misidentified. The results also indicated significant differences in the pupil change responses between the two groups. The nondeviate males consistently dilated to pictures of adult females and the female-aggressing pedophiliacs did not dilate or constrict to the same pictures. Female-aggressing pedophiliacs, on the other hand, most often showed pupil dilation to pictures of immature females, while only half of the nondeviate males showed pupil dilation to the pictures of immature females. It appears, on the basis on this study, that both instruments used have significant value in identifying incarcerated female-aggressing pedophiliacs from other incarcerated but nondeviate males.

This study will report an attempt to investigate nonviolent child-molesting behavior. The primary goal of the study was an attempt to differentiate child molesters from nondeviate males. Differentiation is important, in that, if pedophiliacs can be efficiently detected from "normal" males, then child molesters can be identified without having to catch them "in the act." Diagnosis of a specific sexual deviancy such as child molesting also would make it possible to develop more specific approaches in modifying this behavior. A sexual deviancy scale developed by Marsh, Hilliard, \& Liechti (1955) and pupillometrics (Hess, 1965) were the two instruments used in differentiating the molesters from the nonmolesters. These two instruments were selected because they seemed to be the most relevant based on a review of pertinent research. It was hypothesized that the female-aggressing pedophiliacs would show a higher score on the sexual deviancy scale and would show pupil dilation to slides of young girls and constriction to slides of adult females when compared to the control group. METHOD

The female-aggressing pedophiles in this study consisted of 10 known molesters who had been incarcerated because of their behavior. They had nonviolently abused female children under the age of 12 . "Nonviolent" meant not raping, biting, or cutting the child, but included exposing their genitals, petting the child's genitals, having the child fondle the adult's genitals, etc. The requirement of the female targets being under the age of 12 was made so that the focus of

* Now at Eastern Washington State College, Cheney. Wash. 99004. attention was on those men who had selected obviously physiologically immature sex objects.

A control group of 10 nondeviate males were selected from the same prison population. The controls were selected by randomly choosing prison charts and eliminating those who were sexual offenders and those who had serious visual problems or brain injuries.

Data were then collected on both the experimental and control group in terms of IQ, age, education, and years of imprisonment since the last conviction. All the $S s$ were then interviewed individually and asked to participate in the study. They were told that their cooperation would not directly affect their stay at the prison, but, as is the custom, notations of their participation would be made in their records. The Ss were aware of the confidentiality of the information. Each $\mathbf{S}$ was individually seated at an apparatus similar to Hess's (1965) equipment but using a television camera instead of a 2 -frame/sec $8-\mathrm{mm}$ camera. The following specific instructions were then given, "[S's name], you will be looking at pictures on a screen. Every other slide will be blurred beyond recognition. Do not attempt to determine what is on the blurred slides."

The S was then placed with his head at the eye opening, and then given additional instructions: "At the time you are looking at the slide pictures, we will be taking pictures of your eye to help us measure pupil changes during your observations. It is important that you hold your head as still as possible and also try to keep from blinking. Look around the inside of the box now so that you can become familiar with the apparatus, but do not look around later."
There were seven slides selected that were pertinent to this study. A brief description of these slides can be found in Table 1.

In order to measure just the change in pupil size due to the type of picture shown and to eliminate differences in pupil size due to the different illumination levels, control slides were made by making an unidentifiable blurred picture of the experimental slides. Each control slide was presented just before the experimental slide so that the S's eye adapted to the illumination level.

The slides were then shown to each $S$ in a preselected random order, which was different for each S. Instructions about the control slide and about holding the eye still were included in an effort to reduce problem-solving activity. It has been shown by Beatty \& Kahneman (1966) that memory tasks appear to increase the pupil dilation and, therefore, an attempt should be made to reduce memory work while Ss are viewing the slides.

The experimental and control slides were each presented for $10 \mathrm{sec}$ on a translucent screen by a Continental 55 Slide Projector which had an automatic slide change system. Pictures of the pupil were taken by a Packard Bell television camera with an adjustable lens and recorded on 1-in. television tape. A microphone was placed by the slide projector so that the sounds of the slide being changed could be recorded. This sound helped later in measuring the pupil size at given intervals after the slides were presented.

Pictures of the eyes which had been recorded on video tape were later reproduced on a 24-in. television screen in order to enlarge the pupil

Table 1

Description of Slides

\begin{tabular}{cl}
$\begin{array}{c}\text { Slide } \\
\text { Number }\end{array}$ & \multicolumn{1}{c}{ Description of Slides } \\
\hline 1 & $\begin{array}{l}\text { An attractive adult nude female } \\
\text { lying on her side. }\end{array}$ \\
2 & $\begin{array}{l}\text { An attractive adult nude female. } \\
\text { The female is standing; the upper } \\
\text { torso is visible. }\end{array}$ \\
3 & $\begin{array}{l}\text { An attractive nude adult female } \\
\text { who is lying on a beach with her } \\
\text { breasts visible. }\end{array}$ \\
4 & $\begin{array}{l}\text { A nude female child about } 5 \text { to } 8 \\
\text { years of age lying face down in a } \\
\text { secluded woody area. } \\
5\end{array}$ \\
$\begin{array}{l}\text { A nude female child } 5 \text { to } 8 \text { years } \\
\text { of age sitting facing the viewer. }\end{array}$ \\
$\begin{array}{l}\text { A female child of about } 8 \text { to } 12 \\
\text { years of age dressed in panties and } \\
\text { undershirt. It could be identified } \\
\text { as coming from a mail-order } \\
\text { catalog. } \\
\text { Three female children, age } 3 \text { to } 5 \\
\text { years, dressed in fancy white } \\
\text { dresses. Their backs are toward } \\
\text { the viewer. }\end{array}$ \\
\hline
\end{tabular}


